\title{
A hypothesis regarding how sleep can calibrate neuronal excitability in the central nervous system and thereby offer stability, sensitivity and the best possible cognitive function.
}

\author{
JH Sture Hansson, Stockholm, MD, PhD \\ Retired, former Department of Clinical Neurophysiology, Farsta Stockholm
}

ORCID ID 0000-0003-3383-1217

Email: jh.sture@outlook.com

Phone: $+46(0) 703315317$

Keywords: sleep function, excitability, stability, sensitivity, central nervous system, calibration.

Conflict of interest: none.

Funding: no funding,

\begin{abstract}
The function of sleep in mammal and other vertebrates is one of the great mysteries of biology. Many hypotheses have been proposed, but few of these have made even the slightest attempt to explain the essence of sleep - the uncompromising need for reversible unconsciousness. During sleep, epiphenomena - often of a somatic character - occur, but these cannot explain the core function of sleep. One answer could be hidden in the observations made for long periods of time of the function of the central nervous system (CNS). The CNS is faced with conflicting requirements on stability and excitability. A high level of excitability is desirable, and is also a prerequisite for sensitivity and quick reaction times; however, it can also lead to instability and the risk of feedback, with life-threatening epileptic seizures. Activity-dependent negative feedback in neuronal excitability improves stability in the short term, but not to the degree that is required. A hypothesis is presented here demonstrating how calibration of individual neurons - an activity which occurs only during sleep - can establish the balanced and highest possible excitability while also preserving stability in the CNS. One example of a possible mechanism is the observation of slow oscillations in EEGs made on birds and mammals during slow wave sleep. Calibration to a genetically determined level of excitability could take place in individual neurons during the slow oscillation, so that action potentials are generated during the oscillations "up-phase". This can only take place offline, which explains the need for sleep. The hypothesis can explain phenomena such as the need for unconsciousness during sleep, with the disconnection of sensory stimuli, slow EEG oscillations, the relationship of sleep and epilepsy, age, the effects of sleep on neuronal firing rate and the effects of sleep deprivation and sleep homeostasis. This is with regard primarily to mammals, including humans, but also all other vertebrates.
\end{abstract}




\section{SLEEP: A GENERAL BACKGROUND}

In mammals, sleep is a state of reversible unconsciousness from which the individual is easily awakened, with reduced reactive ability, disconnection of sensory input to the thalamus and cortex and reduction of motor functions. All vertebrates engage in some form of sleep. In mammals and birds, sleep is dominated by non-rapid eye movement sleep (NREM sleep), which includes slow wave sleep (SWS - characterized by an EEG diagram with slow wave activity (SWA) (1) - as well as rapid eye movement sleep (REM sleep) (2) (3). Birds and cetaceans have the ability to sleep unihemispherically (4). Insects such as bees (5) and Drosophila melanogaster (6) (7), mollusks such as cuttlefish and octopus (8) (9), the pond snail (10) and the Cassiopeia jellyfish (11) engage in sleep-like behavior. This shows that sleep has an important, indispensable and fundamental function not only in humans and other mammals but throughout the animal kingdom.

During sleep, the brain's interaction with the external environment is at a low level, and it can be assumed that the individual must pay a price for this state. The absence of consciousness leads to vulnerability to predators and other dangers (12). However, evolution has not been able to eliminate the need for sleep, and even if sleep is expressed in different ways by different species, its core function can be the same. This does not rule out that NREM sleep and REM sleep could have developed independently at various points during the evolutionary process (4).

Other physiological functions outside the nervous system are also affected. Because these functions are not dependent on reversible unconsciousness, they are hardly associated directly with the true reason for sleep, and can thus be considered epiphenomena. Circadian rhythms control not only sleep and rest but also metabolic, immunological and hormonal functions, without these being involved with the true function of sleep.

Consciousness, unconsciousness and sleep are the terms for distinct functional states of brain function, whose outer boundaries are fixed. The human brain has been estimated to contain 86 billion neurons and 85 billion non-neurons (13). Within a limited volume, perception, cognition, memory, 
motor functions, emotional functions, sensitivity, precision and reaction times should be spurred to perform at the highest possible level and balance. Despite general requirements on the conservation of metabolic resources, the human brain is characterized by high metabolism, with up to $20 \%$ of the body's total energy consumption taking place in only $2 \%$ of the volume. Metabolic activity decreases only marginally during sleep. Thus the brain remains active and does not rest during sleep. This has been seen in EEGs registered on the scalp surface. The electrical activity dominated during wakefulness by rhythms of high frequency and low amplitude is transformed during sleep to an activity with low frequency and high amplitude, i.e. SWA within the frequency band of 0.5-5 Hz (Fig. 1). In humans, sleep can be divided into approximately three to six cycles, where each cycle lasts 60 to 90 minutes. Each cycle undergoes a transition period of sleep stages 1-2 and is then dominated by SWA during sleep stage 3, and this is followed by a shorter period of REM sleep. During REM sleep, the surface EEG returns to a pattern similar to that observed during wakefulness.

\section{PREREQUISITES AND CONCEPTS OF SIGNIFICANCE FOR THE HYPOTHESIS}

Evolution has demonstrated an ability to find solutions to complex problems. This is why it makes sense to study evolution as a powerful problem-solver based on drives, details and solutions, and as if evolution has had the ability to behave as a designer. The following presents problems encountered with stability in the neurons in networks as well as with stability within neurons. Examples will be given of how stability can be established with reasonable and existing physiological mechanisms.

Observations will also be provided which more or less provide support for the presented solutions. First and foremost the examples show that solutions are possible. It is possible that evolution has found these solutions, but perhaps more elegant routes have been discovered. Sleep became evolution's contribution to improved function of the nervous system. The solution has offered such significant competitive advantages that it has been incorporated in the brain and nervous system of all more advanced organisms. 
The following review refers primarily to mammals, including humans, but can refer as well to all high-level organisms in the animal kingdom. The pertinent parts of the CNS in mammals are primarily the thalamus and the cortex, which dominate the gray matter.

\section{The nervous system as an alarm system}

In very simplified terms, the nervous system - from low-level to high-level organisms such as mammals - can be perceived as an alarm system with the capability to adapt simultaneously to temporary or permanent changes in the external environment. This ability offers the greatest possible sensitivity and speed without the incidence of false alarms, and this also demands precision and balance. Evolution has adapted the nervous system to each species' external environment. In an extreme situation, the correct and immediate response to stimuli can be the difference between life and death for an individual or offspring. The nervous system must choose - friend or foe, predator or prey, fight or flight - in highly stressful situations. Example of adaptations include advanced vision in birds of prey, the keen sense of smell possessed by dogs and pigs, and the speed and strength of predators and prey animals. Primates, including humans, possess a highly developed cognitive ability. Thus, evolution has driven the nervous system to enable the best performance within a range of areas. Sleep plays an important role here.

\section{Plasticity}

Plasticity is the general term for the adaptation of the brain or ganglia to the external environment, and the foundation for experience, learning, memory and development. One important form of plasticity is Hebbian plasticity, a reinforcement of the synapses which are most active during impulse generation from one neuron to another (14) (15). This form of plasticity is associated with certain forms of memory, such as declarative and procedural memory (16) (17). 


\section{Excitability}

Excitability is a term for both the networks in the nervous system and individual neurons, and can be explored using technical electrophysiological methods such as sensory evoked potentials and magnetic stimulation. A common factor for both these in vivo and non-invasive tests of excitability is that they are more or less dependent on complete networks with excitatory and inhibitory functions.

Transcranial magnetic stimulation activates for example both excitatory and inhibitory neurons. Even though excitability in networks has been studied during sleep, little is known about the excitability of individual, isolated neurons during and after sleep compared with excitability during consciousness (with the exception of a few research efforts (18) (19) (20)).

Contributors to excitability in individual neurons include components such as synaptic strength, which is influenced by synapses, structural conditions, receptors for signaling substances, ion channels etc. (Fig. 2). Another area can be described as non-synaptic excitability with ion channels and structures in dendrites and soma, where the travel of impulses takes place with cumulative graded potentials. A decisive, subsequent component in non-synaptic excitability is intrinsic excitability, which can be determined by the threshold for the electrical excitement of an action potential in axonal initial segments (AIS) (21) (22), consisting largely of voltage-dependent $\mathrm{Na}$, $\mathrm{Ca}$ and $\mathrm{K}$ channels. This threshold is relevant for stimuli with action potentials from synapses or as gap junctions, or through electrical fields, for example in the case of epileptic discharges.

Synaptic scaling and negative feedback in non-synaptic excitability and intrinsic excitability

One mechanism that compensates for the neurons' changing requirements and plasticity's destabilizing influence is synaptic scaling (23) (24), which is most easily described as negative feedback in synaptic strength. Regulation of synaptic strength and non-synaptic excitability can be mediated via the firing rate (FR), even if other mechanisms cannot be ruled out. Variations of FR must be placed in relation to the individual neuron's state of equilibrium FRfb during consciousness, with a characteristic value for each neuron, with a wide range from $0.01 \mathrm{~Hz}$ to $10 \mathrm{~Hz}$ (23). High levels of impulse traffic in the 
neuron reduces synaptic strength in all synapses in the neuron and thus suppresses the impulse traffic through a homeostatic regulation that is mediated by FR (24) (19). Synaptic scaling depends on activity, and locally in the short term, it acts as a stabilizer. The neuron's intrinsic excitability can be regulated in a similar, activity-dependent way with negative feedback (25) (21). Because synaptic scaling can be perceived as having an unclear definition, the more specific terms negative feed-back in synaptic strength (including all the synapses of the neuron) and negative feedback in non-synaptic excitability or intrinsic excitability are sometimes used.

A mechanism for shared bidirectional homeostatic regulation via FR of excitatory synaptic scaling and intrinsic excitability, which is thus not limited only to synaptic scaling, has been shown (26).

One unanswered question is whether regulation of synaptic scaling without connection to nonsynaptic excitability exists in vivo. Earlier findings on synaptic scaling considered only the synaptic contributions to excitability, and the relationship between synaptic scaling and non-synaptic excitability has only recently been noted. For most of the reasoning in this work, the form of this relationship is not important, with the exception of the topic discussed to be discussed later here: the regulation of synaptic strength that follows calibration.

One risk with negative feedback in synaptic strength and non-synaptic excitability is that neurons which seldom need to be active would risk being regulated upward, though it is not clear that the rare activation of a particular neuron depends on excessively low excitability. The following simplified thought experiments demonstrate a need for stability that can resist activity-dependent synaptic scaling and feedback in non-synaptic excitability. Assume that red is the signal for danger. The signal is unusual but is expected to initiate activity in a hypothetical neuron or local network, and it requires a specific response. In the absence of a red signal, the impulse traffic increases as a result of negative feedback in synaptic strength and non-synaptic excitability. The frequency of false red signals - with the possibly undesirable reaction this could cause - would thus increase. Over a longer period of 
time, a fixed level of excitability would guarantee stable and balanced function, even in the case of these potentially important, but perhaps seldom active neurons (12). The opposite can also be possible for very active neurons. Below, a solution for this is shown: the resetting of the neuron to its genetically predetermined, stable excitability through calibration.

\section{The balance between excitability and stability}

Instability is built into the function of a collection of neurons in a network (23). A nerve impulse normally generates a wave of impulses that move from node to node in the axon. An impulse from a neuron can generate impulses in other neurons through synapses, gap junctions and ephaptic transfer. In this system, an uncontrollable chain reaction or feedback can easily occur, and if excitability in the neuron or network is too high, the worst-case scenario is that this will spark an epileptic discharge.

Electroconvulsive therapy (ECT) is used in psychiatry as a treatment for psychoses and mood disorders (27). Electrodes placed on the surface of the skull create electrical currents in the brain which induce reactions similar to epileptic seizures. Anesthesia and muscle relaxants reduce the risk of injury. The placements of electrodes and other strategies vary, but remarkably, it seems that seizures can always be induced (27). The potentials for epileptic discharges induced through ECT are not limited to humans; it has been shown that a model for ECT exists in rats (28) (29). This can lead to important conclusions. The level of excitability in individual neurons and the cortex is always sufficiently high for spreading of epileptic seizures. Nevertheless, epileptic seizures rarely occur spontaneously in a healthy brain. Exceptions exist, for example in the case of alcohol abstinence, sleep deprivation, febrile convulsions in children and treatment with certain drugs. In these cases, it is plausible that the general level of excitability in the neurons and networks is elevated.

Fig. 3 attempts to illustrate variants of excitability in cortical neurons with regard to best function, so that some conclusions may be drawn from studies of ECT.

A: generally extensive low excitability would prevent ECT; therefore, this case can be excluded. 
B: a relatively high, but uniformly distributed excitability can be consistent with the observations based on ECT. The level of excitability is always sufficiently high to allow spreading of epileptic discharges which have been induced.

$\mathrm{C}$ : a medium-high level of excitability as in $\mathrm{B}$, but with greater spreading of excitability, as in the case of neurons with unnecessarily low excitability together with neurons with a dangerously high level of excitability. The latter can be expected to involve greater risk for false-positive reactions and spontaneous epileptic discharges; this alternative is thus unfavorable.

Other variants can also be tested using this intuitive model. Alternative B remains as the most advantageous.

In addition to the animal model for ECT in rats, veterinary medicine has noted epilepsy-like seizures in such varied species as dogs, cats, horses, guinea pigs, birds and reptiles. Single or recurring epileptic seizures are always a threat to the life and health of an individual or species, and therefore must be avoided at all costs. The example of ECT leads to the conclusion that it despite this is advantageous to the function of many individuals of many animal species that excitability in networks and individual neurons is a constant and difficult balancing act between maintaining the highest possible level and yet ensuring that the threshold for spontaneous epileptic discharges is not exceeded.

Prior to initiation of epileptic discharges, inhibitory neurons exert a suppressive effect through synapses. However, a subsequent spreading can be non-synaptic, where the mechanism can also be mediated via electrical field effects, as shown in the hippocampus (30) (31); paradoxically, this would possibly mean that inhibitory neurons with high excitability could also contribute. Theoretically, therefore, it is not certain that high excitability in inhibitory neurons can prevent the spreading of epileptic impulses. This could mean that a regulation of network excitability solely through the balancing of inhibitory neurons against excitatory neurons would only succeed to certain degree before seizures spread. This regulation would thus be unreliable. 
For this reason, evolution has developed an acceptably reliable level of excitability in individual neurons. This level is as high as possible but still below the level at which unprovoked adverse reactions and epileptic seizures can occur (Fig. 4A). This means that neuronal excitability maintains a high, evenly distributed level, and in the absence of provocation, excitability will not induce potentially life-threatening, general epileptic seizures (Fig. 4B). However, despite this, seizures can occur in the case of illness or other causes such as ECT (Fig. 4C). This presumes a well-considered regulation of excitability in all neurons within all regions of the cortex.

If stability is to be maintained, the excitability of all individual neurons must have an upper limit which is determined by the requirement that

- unprovoked seizures must be avoided

- saturation of the neuron, which even at moderate levels of stimulation would react with maximum effect (21), needs to be avoided

- excessively high sensitivity can result in false-positive reactions Excessively low levels of excitability must also be avoided, because they can result in

- slow reaction times

- false negative reactions

- general sluggishness and reduced cognitive function

The level of excitability that incurs risk for seizures and false-positive reactions cannot be directly identified in a simple way, for example as body temperature can be continuously regulated through detection of temperature. Excessively high temperatures are detected by sensors and are lowered, just as excessively low temperatures are raised. Seizures and false-positive reactions, on the other hand, occur without any prior warning signs, just as excessively low excitability with accompanying lack of performance does not offer detectable signals. Only the species' experiences over time, inherited via evolution, can determine the optimal level of excitability - and this level can differ for the various parts of the neuronal networks. 
At the same time that excitability is to remain within these stringent limits, the conditions for stability are continually challenged by constant changes taking place in the brain (32):

- growth and pruning of neurons and changes in neuron geometry

- turnover of ion channels

- neuromodulatory changes

- changes related to aging

- activity-dependent influences such as Hebbian plasticity and negative feedback in excitability

Multiple neurons can be part of a chain for one and the same function. High excitability in a neuron in a chain that is part of a network cannot be compensated by low excitability in another neuron. This kind of regulation would lead to suboptimal function in both neurons.

It can be assumed that the excitability in neurons that are active during consciousness can be shifted in one or the other direction from the optimal level, with negative consequences. A level that is stable despite variations in the external and internal conditions of the nervous system requires regular resets through calibration that includes excitatory and possibly also inhibitory neurons.

\section{THE HYPOTHESIS}

Highly developed species in the animal kingdom - mammals and others - can be described by the argument below. All neurons in the CNS, regardless of whether they are excitatory (with for example transmitter substances such as glutamate or acetylcholine), and thus stimulating the postsynaptic neuron, or inhibitory (such as GABA-erga), and thus suppressing the postsynaptic neuron, must contribute to the best possible functions with respect to impulse creation.

"Calibration" is a technical term referring to checking and adjustment against a set standard; in this hypothesis, the standard is a genetically predetermined and fixed level of excitability, regardless of the activity. No neuron can be allowed to steal space and metabolic energy without contribution to the best function, or worse, inducing an incorrect function. The goal of the best possible function is highly 
dependent on stable, predetermined and well-defined excitability in individual neurons. The guarantor of this excitability is calibration. Calibration cannot take place during normal activity, and this explains the need for sleep. Activity-dependent negative feedback maintains temporary stability during periods of wakefulness between calibrating sleep periods. In addition to the mandatory requirement of sleep, it can be assumed that calibration has many physiological mechanisms.

The following describes a proposal regarding the physiological mechanisms for calibration, with the purpose of demonstrating that calibration is possible, and the consequences that this could have. These mechanisms should be understood as examples, and thus there may be other mechanisms at work here.

Every credible sleep hypothesis must explain why reversible unconsciousness is a necessary and central condition (12). It is not sufficient to say that unconsciousness is one of many equivalent factors that merely facilitate the function of sleep. In that case, evolution would have been able to eliminate sleep for one or more species or individuals.

A calibration of neuronal non-synaptic excitability requires a predictable, stable environment without external influences in the form of uncontrollable impulses with origins in the sensory organs. The condition can be fulfilled through a disconnection of the brain when a) the individual seeks out an undisturbed environment through sleep and b) the afferent impulse traffic is reduced on one or more levels and cognitive and other functions (including the synaptic function) are suppressed in the brain and finally unconsciousness. The impulse traffic generated during calibration would not only prevent meaningful function but would also lead to chaotic, damaging function; therefore, motor functions must be reduced.

\section{SWA}

SWA is most intensive at the beginning of SWS. In mammals, after they experience sleep deprivation, the time for SWS (sleep homeostasis) is extended (33). Electrodes placed deep in the brain or 
intracellularly in thalamic or cortical pyramid cells have shown that the polarization of the cell membrane during SWA switches between an up and a down state, in which "up" is a depolarized, excitable state close to the cell membrane's resting potential and "down" is a hyperpolarized, nonexcitable state that is synchronous across groups of neurons (34). Despite thorough and detailed knowledge of the membrane characteristics of neurons and networks in vitro and in vivo during SWS, and in trials using anesthetized animals, consensus is lacking regarding the slow oscillations (34). Their function has been called a mystery; however, the notion that they play a role in the creation of memory has been proposed (35), as has their participation in a normalization of synapse strength (36). How these oscillations are generated is unclear (37). The oscillations are characterized by a square wave with an up- and down-phase with a frequency of $<1 \mathrm{~Hz}$. The shifts from up to down and down to up can be described as rapidly decreasing functions, while the up- and down-phases have a stable plateau shape (37) (38). The down-phase has a shorter duration (19) (39). During the up-phase, discharges of action potentials occur (34). It has recently been shown that in the frontal pyramid cells of rats, the statistical distribution of FR decreases during SWS (19), with fast-firing pyramid cells having lower FR while slow-firing pyramid cells have higher FR. The slow oscillation could be part of a mechanism for calibration.

\section{The calibration mechanism for non-synaptic excitability and SWA}

The following mechanism for the generation of action potentials and calibration during SWA should be understood as a theoretical proposal. During the down-phase with hyperpolarization, synchronously within larger regions, significant stoppage of circulating impulse traffic takes place in the region. The inactivation of $\mathrm{Na}$ channels - and during the up-phase this involves a significant number of $\mathrm{Na}$ channels (40) - is halted. All Na channels in relevant neurons in the AIS thus become available for activation, and non-synaptic excitability increases. As a result, during the transition to the up-phase, 
trains of action potentials are created. The down-phase can also contribute, based on hyperpolarization, to the stabilization of the membrane potentials of the up-phase.

If the oscillation's up-phase close to the resting phase of the membrane potential encounters a high threshold and the accompanying excessively low non-synaptic excitability that does not lead to action potentials or action potentials with low FR, this will lead - via negative feedback in nonsynaptic excitability - to a slight decrease of the threshold for each period of the up-phase, and nonsynaptic excitability will increase until action potentials are generated with increasingly higher FR until the FRcal (which can but is not required to be identical with FRfb) is reached. If in a corresponding manner the oscillation's up-phase encounters a low threshold and high non-synaptic excitability, which results in excessively high FR relative to FRcal, there will be a successive increase of the threshold level and downward regulation of excitability via negative feedback. Gradually the threshold level will be calibrated to a level adapted to the polarization of the up-phase, with the neuron's FRcal as the target for regulation. The entire neuron participates in impulse generation, but the decisive factor for this feedback with regulation of excitability (in addition to dendrites and soma) is found in the AIS (41) (42). Growth and regeneration of voltage-sensitive Na channels, K channels and Ca channels can offer such mechanisms (43) (44). On a cellular and molecular level, there are many examples of mechanisms that enable regulation of both synapses and intrinsic excitability. For summary reviews, see Krueger/Obal (12) or Nelson/Turrigiano (45). Regardless of the functions among these that are active in a calibration, the main principles of the hypothesis still apply. Therefore, with calibration during slow oscillations, a genetically predetermined, stable threshold is created for impulse generation in the neuron. Inhibitory neurons can also require calibration to maintain a balance with respect to excitatory neurons (46) (47).

Neuronal excitability is determined by non-synaptic excitability as well as synaptic strength. It is possible that in this first step, calibration with slow oscillations does not affect synaptic functions and concerns primarily non-synaptic excitability. 
The fact that SWA can play a role in calibration does not rule out another type of calibration in limited neuron populations or regions of the brain during NREM or REM sleep. This has not been observed, but this could be because they perhaps do not generate equally noticeable field effects in EEGs, or clear variations in membrane potentials. The cerebellum, hippocampus and basal ganglia contain neurons that can be stabilized to good advantage with calibration, just as other sleep rhythms (such as sleep spindles) could have a calibrating function. The existence of other mechanisms for calibration in other species could also explain why SWA has not been observed in species other than birds and mammals. The lack of SWA in animals such as reptiles and Drosophila melanogaster indicates that they are equipped with other mechanisms for sleep calibration.

REM sleep is also characterized by reversible unconsciousness. As in the case of SWS, it is difficult to find another credible cause than function control and calibration, but in REM sleep, SWA with impulses of an up-down character - and with the distinct transitions that should thus be observable - is absent. REM sleep can also be part of the secondary regulation of synaptic strength, as discussed below.

\section{A proposed mechanism for secondary regulation of synaptic strength based on non-synaptic}

\section{excitability}

The calibration of non-synaptic excitability could have a secondary purpose of stabilizing synaptic strength, which is included in the neuron's total excitability; this is shown in the following example with a stepwise, iterative process. The example attempts to show that a solution is possible and that nature can have discovered this or similar solutions. Feedback in synaptic strength, in common with non-synaptic excitability, can lead to a specific synaptic strength via resetting of FR, whose desired state of equilibrium FRfb has been altered during the calibration of non-synaptic excitability. As a result, both synaptic strength and non-synaptic excitability will be able to reach a single, unique, stable equilibrium, and be bound to each other. 
This secondary feedback regulation of synaptic strength and non-synaptic excitability can take place during non-SWS, i.e. during REM sleep or light sleep. In the absence of sensory signal input, spontaneous, post-synaptic excitatory and inhibitory miniature potentials have noise-like characteristics, quite stationary and unchanged from calibration to calibration (46) (38). In the first step after calibration, the secondary feedback regulation will once again shift non-synaptic excitability from the level of FRcal, which was the target during calibration, to the level of FRfb (Fig. 5a, legend in Fig. 5b and the Appendix). The calibration with SWA can then return with additional steps toward ever smaller deviations of FR from the desired levels of FRfb and FRcal in an iterative process that switches between SWS and non-SWS. A calibration of the neuron's total excitability will thus be facilitated, for example by human sleep architecture, by a cyclical process where SWS is followed by REM sleep and light sleep. The sleep architecture of dogs (48), cats (49) and rats (50) with its rapid and frequent transitions between SWS and non-SWS can also facilitate the calibration of neuronal excitability.

How feedback regulation that is controlled by FR is divided between synaptic strength and nonsynaptic excitability is not easily determined and has no clear solution, with the exception of the conclusions that can be drawn based on the presumed iterative process. If the secondary feedback process included only synaptic strength without influencing non-synaptic excitability, the relationship between synaptic strength and non-synaptic excitability would be fixed after only one period of nonSWS and an iterative process would be unnecessary. If the process is limited to allowing synaptic strength to dominate over non-synaptic excitability, the process will require fewer loops.

The alternative where synaptic strength together with non-synaptic excitability would only stabilize FR to FRfb, without the prior calibration of non-synaptic excitability, would hypothetically lead to a large number of alternative solutions with varying relationships between non-synaptic excitability and synaptic strength. High synaptic strength would thus seem to be able to be compensated with a low non-synaptic excitability and a seesaw-like state would be created. High 
synaptic strength would be unstable and would also result in poor resource management with respect to volume requirements and energy consumption. Above all, the solution using the alternative of low synaptic strength and high non-synaptic excitability would be unstable. In summary, an undetermined relationship between synaptic strength and non-synaptic excitability would result in unstable conditions with a risk of epileptic discharges as well as wasted volume and energy. Thus, a stable solution is delivered by the calibration of non-synaptic excitability to FRcal followed by the iterative process with negative feedback of synaptic strength together with non-synaptic excitability to FRfb. This way, calibration can include both non-synaptic excitability and secondary synaptic strength - and thereby the neuron's total excitability.

\section{White matter needs no calibration}

In addition to dendrite trees with synapses, soma, axon hillock and AIS, the neuron contains axons with myelin sheaths (Fig. 2). White matter, whose volume makes up approximately $50 \%$ of the human brain, consists primarily of axons. The axon diameter and myelin sheath thickness are configured to maximize impulse speed; therefore, they are structurally and genetically predetermined. A large part of the energy transformation takes place in nodes with voltage-dependent $\mathrm{Na}$ and $\mathrm{K}$ channels, but the energy consumption in these channels is only about $0.5 \%$ of the brain's total energy consumption

(47). The impulses can therefore travel with a high safety factor, without significant need of adaptation to metabolic requirements and limited volume. All this means that the node threshold level can vary without notable effects on function, and that white matter with its axons does not need calibration.

\section{OBSERVATIONS THAT CAN SUPPORT THE HYPOTHESIS}

\section{Effects of sleep deprivation}

Sleep deprivation leads to increased cortical excitability, measured through median-nerve stimulation and transcranial magnetic stimulation (51) (52). Sleep calibration restores excitability in individual 
excitatory and perhaps inhibitory neurons and thus returns networks to their original levels. In addition, sleep deprivation has been shown to diminish cognitive function in humans (53). Sleep deprivation can be expected to lead to reduced balance, with increased and/or decreased excitability in excitatory or inhibitory neurons. This is why sleep with calibration restores excitability in these components to the optimal level and thus improves cognitive function.

Partial or total sleep deprivation results in general increased reaction times (54) (55). This is contradictory according to the observations above regarding increased excitability that has been observed in cortical networks, but it would be consistent with reduced excitability in certain neurons after sleep deprivation, in analogy with the notion that reduced excitability is associated with increased latency from stimulus to action potentials in neurons in tissue cultures (56). Sleep with calibration restores reaction times. However, increased reaction times after sleep deprivation are difficult to combine with generally increased excitability, which is one of the prerequisites for Synaptic Homeostasis Hypothesis (SHY, see below).

Sleep deprivation results in unstable and unpredictable behavior patterns (54), not just the lack of response to stimulation (false-negative response) and response without stimuli (false-positive response). This could be consistent with the presence of uncalibrated neurons with randomly increased or decreased excitability.

Sleep deprivation can induce clinical seizures or epileptiform interictal activity in EEGs (57). Possible causes could be uncalibrated excitatory neurons with high excitability or inhibitory neurons with low excitability (47) (58) (59). Nor can inhibitory neurons with high excitability be excluded in this content. Calibration to optimal excitability reduces this risk.

\section{Sleep homeostasis}

Long periods of wakefulness can be expected to lead to greater deviations from the optimal level of excitability. The duration of SWS has been shown to increase after sleep deprivation, which could be 
explained by larger deviations prior to calibration (60) (61). The time for resetting this excitability to a suitable level therefore increases, and this is reflected in the duration of sleep after longer periods of wakefulness or sleep deprivation.

Age-related variations in sleep

During growth and development, the brain changes the neuronal environment very quickly, and this places greater requirements on calibration. Young individuals sleep longer and with more SWA, while older individual sleep less and with less SWA.

\section{Memory consolidation}

Under certain circumstances, NREM sleep can affect plasticity. During the critical period for visual development in cats, sleep strengthens the synaptic remodeling that takes place after monocular deprivation (MD) (62). This finding can be considered a typical example of memory consolidation during sleep.

The following arguments could be applied to MD, but also to other events that lead to plasticity, with either increased or decreased neuronal excitability or impulse traffic. Excitability reduced by plasticity in the synapses in question and the resulting decrease in the neuron's impulse traffic would lead to negative feedback in the conscious state, which counteracts the effect in these neurons with some lessening of the decrease in excitability as a result (neuronal response in Fig. 6). According to this hypothesis, a subsequent calibration during sleep with resetting of excitability - which would decrease - would thus counteract the effect of feedback in excitability and partially restore the effect of plasticity. The corresponding argument can be made for events with increased excitability, which with increased impulse traffic in the neuron in question is counteracted by feedback in excitability. Calibration restores excitability, and thus appears to have strengthened the effect of plasticity. Both of 
these variants of calibration, where the threshold is regulated up or down, partially restore the effect of plasticity and could contribute to a result that resembles consolidation during sleep.

\section{FR homeostasis inhibited during sleep}

Disturbances in the form of visual deprivation affect FR in rats, first as a result of plasticity with a reduction in FR, which after several days returns to the value that is characteristic for the neuron (18). During sleep cycles with a mean duration of 18 minutes, this phase of restoration is halted at plateaulike levels that neither increase nor decrease to any significant degree. One interpretation of these findings would be that the calibration, which would thus make a negative contribution to FR, would work in an opposite, balancing direction against feedback in synaptic strength and non-synaptic excitability - which would be expected to result in increased FR during these cycles.

\section{Reduced spreading of FR during sleep}

The spreading of FR in pyramid cells in rats decreases during sleep (19). Fast-rate firing pyramid cells reduce their FR during sleep, while slow-rate firing cells increase their FR. This can be interpreted as a general reduction or increase of FR, based on the pyramid cells' designation as fast- or slow-firing. Another interpretation based on this hypothesis is that wakefulness results in spreading of FR in individual pyramid cells from the original FRfb, Fig. 7.

It can be shown that

$\mathrm{FR}^{2}=\mathrm{FRb}^{2}+\mathrm{FR}_{\mathrm{w}}{ }^{2}$ where

$\mathrm{FR}_{\mathrm{a}}{ }^{2}$ is the variance in FR in the entire population of pyramid cells after a period of wakefulness $\mathrm{FR}_{\mathrm{b}}{ }^{2}$ is the variance in $\mathrm{FR}$ in the entire population of pyramid cells before a period of wakefulness and $\mathrm{FRW}_{\mathrm{W}}{ }^{2}$ is the variance with additional spreading in individual pyramid cells that takes place during the wakeful period. 
This results in a picture of generally increased spreading of FR during wakefulness with a shifting of the distribution graph tails with fast-rate firing toward an even faster rate and slow-rate firing toward a slower rate. During sleep, the spreading decreases once again.

\section{COMPARISONS WITH EARLIER HYPOTHESES}

This section describes a limited selection of earlier sleep hypotheses.

- The Null Hypothesis - sleep occurs because there is no other meaningful activity (63) (64).

- During sleep, metabolic products are released from nerve cells (65).

Neither of these hypotheses or similar ones can explain the absolute need for reversible unconsciousness.

- Memory retention and consolidation have been observed to benefit from sleep, and this has been considered the best explanation for the need for sleep (66).

The opposition to this argument is that memory processes are initiated by experiences gained during consciousness. There are no fundamental or sufficiently strong reasons that consolidation would require unconsciousness, and that this process could not take place during consciousness. Therefore, memory consolidation should be understood as an epiphenomenon, and evolution should have been able to eliminate the need for unconsciousness, unless sleep exists for another reason.

- A hypothesis for the function of sleep, SHY, is particularly interesting because it is based on the need for stability in the brain, and builds on the mechanisms that resemble synaptic scaling and the requirement on the reversible unconsciousness offered by sleep (64) (67) (68). The hypothesis is based on the notion that as a result of Hebbian plasticity and accumulated experiences, synaptic strength generally increases during consciousness. Maintaining stability would require downward regulation of synaptic strength with synaptic downscaling during sleep, which would lead to the normalization of synaptic strength at a lower level. This hypothesis has met with criticism (69) (70). 
Like the hypothesis presented here, SHY is based on the demand for stability in neuronal networks, and arrives at least partially at the same conclusion: sleep stabilizes. However, the two hypotheses then diverge. The difference is that this hypothesis assumes that negative feedback in neuronal excitability takes place during consciousness, and that a subsequent calibration to genetically predetermined levels can both increase and decrease excitability in individual neurons. Calibration is needed not only to counteract activity-dependent negative feedback in non-synaptic excitability, but also to counteract other changes in the nervous system.

One similarity is that both hypotheses include slow oscillations as part of a possible mechanism for regulation, with downward adjustment of synaptic strength in SHY and up- or downscaling of neuronal excitability in this hypothesis. In connection with SHY, computer simulation has shown that under certain circumstances, downscaling can result in a bistable function with SWA-like up- and down-states, and that this could actively contribute to "renormalization" (36). In this hypothesis, SWA is assigned as active role in the regulation of non-synaptic excitability.

The foundational principle of SHY - a general downscaling during sleep - is an activity that should be able to take place simultaneously with Hebbian plasticity, which operates during consciousness. It is difficult to find counterarguments to this cited reference (69). If so, the need for downscaling during sleep would have been able to be eliminated with the help of evolution.

Nevertheless, sleep is still around, and it clearly has another important function that cannot be sufficiently explained by SHY and downscaling. In addition, it has recently been demonstrated that sleep achieves both upward and downward regulation of FR, and thus excitability (19).

\section{CONCLUSIONS}

Hypothetically, a nervous system could also function without calibration during sleep, but at the cost of diminished and less reliable function and/or greater risk for uncontrolled feedback, i.e., epileptic discharges; however, evolution has clearly abandoned this alternative. 
A basic dilemma for a complex central nervous system is that stability is counter to excitability and flexibility. Instability resulting from sleep deprivation creates a risk of uncontrolled feedback as in the case of epileptic seizures, but can also manifest itself as an unpredictable function. Positive feedback such as Hebbian plasticity contributes to instability. Negative feedback provides only a shortterm solution to the stability problem, and can create other problems, for example in the case of unusual events.

The primary hypothesis - that the function of sleep is to enable calibration of individual neurons in a central nervous system - is founded on the circumstances that can be observed in a close examination of ECT results; excitability in the cortex is higher than would be possible without fine, precision adjustment of excitability in individual neurons. In addition, the very existence of sleep with temporary unconsciousness lacks a plausible explanation. The hypothesis finds more or less support in the previously published observation cited here:

The existence of a notable phenomenon such as SWA has not been clearly explained, but it could have a part to play in calibration.

The risk of epileptic seizures and unforeseen reactions support the notion of increased instability resulting from sleep deprivation, but these effects are counteracted by sleep with calibration, which also stabilizes.

A longer period of wakefulness entails greater deviations from stable excitability, which results in the need for longer calibration times (sleep homeostasis).

Neurons or networks that are seldom active but important cannot be stabilized with feedback, but calibration can solve this problem.

Calibration can only take place offline, and this requires reversible unconsciousness with disconnection from muscular activity.

Sleep architecture with cyclical processes can be consistent with an iterative process based on calibration of intrinsic excitability that extends stability in the neuron to synaptic strength. 
Rapid changes in neurons and the environment of young individuals threaten stability. In young individuals, calibration and sleep require more time.

Under certain circumstances, calibration during sleep could offer results that (at least superficially) resemble memory consolidation.

Calibration of excitability in individual neurons could explain how the spreading of FR during consciousness in rats is restored during sleep.

Sleep resets evolution's genetic memory tracks that have developed of long periods of time, after the less permanent experiences gained during consciousness. During consciousness, flexibility with plasticity is the order of the day, modified by negative feedback in excitability. During sleep, stability is ensured with calibration of excitability.

The calibration process can be compared to the tuning of instruments in an orchestra. All the instruments must be well tuned. The slightest dissonance can ruin the whole. Tuning must be carried out on individual instruments, and cannot be done during the performance.

Knowledge about the function of sleep is essential in human medicine, and sleep is an important factor in conditions such as Alzheimer's disease (71), schizophrenia (72), depression (73) (74), fibromyalgia (75), headaches (76) and pain from rheumatoid arthritis (77), to name only a few. The understanding of the brain cannot be complete without an understanding of the function of sleep.

\section{APPENDIX A}

A model for calculating how synaptic strength can be related to non-synaptic excitability

The model is simplified to provide an intuitive understanding of the mechanisms in synaptic scaling in non-synaptic excitability as illustrated in Fig. 5.

Generally accepted definitions of synaptic strength and intrinsic excitability or the somewhat broader concept of non-synaptic excitability are lacking. The definitions have been selected to provide the clearest and most correct model possible. 
Synaptic strength $(S)$ can be related to amplitude in miniature excitatory postsynaptic potentials (mEPSC) with the dimension current and the measurement value $i$ (78). The measurement value $S$ for the total synaptic strength in question is defined here as

$S=s \times i$

where $s$ is a constant dependent on the number of synapses and structural and other factors in dendrites and soma before the impulses initiate action potentials in axon hillock.

Non-synaptic excitability $(N)$ and threshold level are two different ways to describe the same function. The concept of threshold level is not a simple one, but this argument is based on an excitability primarily in AIS, stemming from synapses, which yields $\mathrm{FR}=\mathrm{FR} \mathrm{fb}_{\mathrm{fb}}$. With the dimension of the current measurement value, the threshold at $\mathrm{FR}=\mathrm{FR}_{\mathrm{fb}}$ is $t$.

Here, the inverted threshold value has been chosen as a definition for non-synaptic excitability $N=1 / t$

If the neuron's synapses are to collectively induce action potentials with FR with the measurement value $\mathrm{FR}_{\mathrm{fb}}$, the depolarizing current from the synapses must be equal to the non-synaptic threshold level $\left(\mathrm{FR}_{\mathrm{fb}}\right)$. This can be expressed as:

$s \times i=t \quad$ or $\quad s \times i \times\left(\frac{1}{t}\right)=1$

i.e. [synaptic strength]x[non-synaptic excitability $]=1$ or

$S \times N=1$

can also be expressed as

$\log S+\log N=0$

If $\log S+\log N=0$, then the neuron is at a balanced level of excitability for the generation of action potentials with $\mathrm{FR}=\mathrm{FR}_{\mathrm{fb}}$

$\log S+\log N>0$ yields $\mathrm{FR}>\mathrm{FR}_{\mathrm{fb}}$

$\log S+\log N<0$ yields $\mathrm{FR}<\mathrm{FR}_{\mathrm{fb}}$ 
Via a feedback loop, FR regulates synapse strength and intrinsic excitability (26) based on a connected mechanism.

In the feedback loop, if

$\mathrm{FR}>\mathrm{FR}_{\mathrm{fb}}$ the mechanism shall result in the mutual reduction of $\log \mathrm{S}+\log \mathrm{N}$;

$\mathrm{FR}<\mathrm{FR}_{\mathrm{fb}}$ the mechanism shall result in the mutual increase of $\log \mathrm{S}+\log \mathrm{N}$.

In the loop FR successively approaches $\mathrm{FR}_{\mathrm{fb}}$, see Fig. 5. 


\section{REFERENCES}

1. Steriade M, McCormick DA, Sejnowski TJ. Thalamocortical oscillations in the sleeping and aroused brain. Science. 1993;262:679-85.

2. Mignot E. Why We Sleep: The Temporal Organization of Recovery. PLoS Biol. 2008;6(4):e106.

3. Rattenborg NC. Evolution of slow-wave sleep and palliopallial connectivity in mammals and birds: A hypothesis. Brain Res Bull. 2006;69(1):20-9.

4. Rattenborg NC, Amlaner CJ, Lima SL. Behavioral, neurophysiological and evolutionary perspectives on unihemispheric sleep. Neurosci Biobehav Rev. 2000;24(8):817-842.

5. Kaiser W, Steiner-Kaiser J. Neuronal correlates of sleep, wakefulness and arousal in a diurnal insect. Nature. 1983;1983:707-9.

6. Nitz DA, van Swinderen B, Tononi G, Greenspan RJ. Electrophysiological correlates of rest and activity in Drosophila melanogaster. Curr Biol. 2002;12(22):1934-1940.

7. Hendricks JC, Finn SM, Panckeri KA, Chavkin J, Williams JA, Sehgal A, et al. Rest in Drosophila is a sleep-like state. Neuron. 2000;25(1):129-138.

8. Frank MG, Waldrop RH, Dumoulin M, Aton S, Boal JG. A Preliminary Analysis of Sleep-Like States in the Cuttlefish Sepia officinalis. Balaban E, editor. PLoS ONE. 2012;7(6):e38125.

9. Harliwich DD. Behavioural aspects of the New Zealand octopus Pinnoctopus cordiformis: acclimation, sleep deprivation and responses to video stimuli [thesis]. University of Canterbury; 2011.

10. Stephenson R, Lewis V. Behavioural evidence for a sleep-like quiescent state in a pulmonate mollusc, Lymnaea stagnalis (Linnaeus). Journal of Experimental Biology. 2011;214:747-56. 
11. Nath RD, Bedbrook CN, Abrams MJ, Basinger T, Bois JS, Prober DA, et al. The Jellyfish Cassiopea Exhibits a Sleep-like State. Curr Biol. 2017 Oct;27(19):2984-2990.e3.

12. Krueger JM, Obal Jr F. Sleep function. Frontiers in Bioscience. 2003 May 1;8:d511-519.

13. Herculano-Houzel S. The human brain in numbers: a linearly scaled-up primate brain. Front Hum Neurosci [Internet]. 2009 [cited 2017 Feb 7];3. Available from: http://journal.frontiersin.org/article/10.3389/neuro.09.031.2009/abstract

14. Markram H, Lübke J, Frotscher M, Sakmann B. Regulation of synaptic efficacy by coincidence of postsynaptic APs and EPSPs. Science. 1997;275(5297):213-215.

15. Magee JC, Johnston D. A Synaptically Controlled, Associative Signal for Hebbian Plasticity in Hippocampal Neurons. Science. 1997;1997(275):209-13.

16. Diekelmann S, Born J. The memory function of sleep. Nat Rev Neurosci. 2010;11:114-26.

17. Ellenbogen JM, Hulbert JC, Stickgold R, Dinges DF, Thompson-Schill SL. Interfering with Theories of Sleep and Memory: Sleep, Declarative Memory, and Associative Interference. Curr Biol. 2006;16(13):1290-4.

18. Hengen KB, Torrado Pacheco A, McGregor JN, Van Hooser SD, Turrigiano GG. Neuronal Firing Rate Homeostasis Is Inhibited by Sleep and Promoted by Wake. Cell. 2016 Mar;165(1):180-91.

19. Watson BO, Levenstein D, Greene JP, Gelinas JN, Buzsáki G. Network Homeostasis and State Dynamics of Neocortical Sleep. Neuron. 2016 May;90(4):839-52.

20. Miyawaki H, Diba K. Regulation of Hippocampal Firing by Network Oscillations during Sleep. Curr Biol. 2016 Apr;26(7):893-902. 
21. Schulz DJ. Plasticity and stability in neuronal output via changes in intrinsic excitability: it's what's inside that counts. J Exp Biol. 2006;209(24):4821-7.

22. D'Angelo E. Homeostasis of intrinsic excitability: making the point. J Physiol. 2010;588(6):9012.

23. Turrigiano GG. Homeostatic plasticity in neuronal networks: the more things change, the more they stay the same. Trends Neurosci. 1999;22(5):221-227.

24. Turrigiano GG. The Self-Tuning Neuron: Synaptic Scaling of Excitatory Synapses. Cell. 2008 Oct;135(3):422-35.

25. Desai NS. Homeostatic plasticity in the CNS: synaptic and intrinsic forms. J Physiol-Paris. 2003;97(4-6):391-402.

26. Joseph A, Turrigiano GG. All for One But Not One for All: Excitatory Synaptic Scaling and Intrinsic Excitability Are Coregulated by CaMKIV, Whereas Inhibitory Synaptic Scaling Is Under Independent Control. J Neurosci. 2017 Jul 12;37(28):6778-85.

27. van Waarde JA, Verwey B, van der Mast RC. Meta-analysis of initial seizure thresholds in electroconvulsive therapy. Eur Arch Psychiatry Clin Neurosci. 2009 Dec;259(8):467-74.

28. Porsolt RD, Le Pichon M, Jalfre M. Depression: a new animal model sensitive to antidepressant treatments. Nature. 1977(Vol. 266):730-2.

29. Li B, Suemaru K, Cui R, Kitamura Y, Gomita Y, Araki H. Repeated electroconvulsive stimuli increase brain-derived neurotrophic factor in ACTH-treated rats. Eur J Pharmacol. 2006 Jan;529(1-3):114-21. 
30. Zhang M, Ladas TP, Qiu C, Shivacharan RS, Gonzalez-Reyes LE, Durand DM. Propagation of Epileptiform Activity Can Be Independent of Synaptic Transmission, Gap Junctions, or Diffusion and Is Consistent with Electrical Field Transmission. J Neurosci. 2014 Jan 22;34(4):1409-19.

31. Qiu C, Shivacharan RS, Zhang M, Durand DM. Can Neural Activity Propagate by Endogenous Electrical Field? J Neurosci. 2015 Dec 2;35(48):15800-11.

32. Marder E, Prinz AA. Current compensation in neuronal homeostasis. Neuron. 2003;37(1):2-3.

33. Dijk D-J. EEG slow waves and sleep spindles: windows on the sleeping brain. Behavioural Brain Research. 1995;1995(69):109-16.

34. Neske GT. The Slow Oscillation in Cortical and Thalamic Networks: Mechanisms and Functions. Front Neural Circuits. 2016 Jan 14;9(Article 88):1-25.

35. Timofeev I, Bazhenov M. Mechanisms and biological role of thalamocortical oscillations. In: Columbus FH, editor. Trends in Chronobiology Research. New York: Nova Science Publishers; 2006. p. $1-47$.

36. Olcese U, Esser SK, Tononi G. Sleep and Synaptic Renormalization: A Computational Study. J Neurophysiol. 2010 Dec 1;104(6):3476-93.

37. Wilson MT, Barry M, Reynolds JNJ, Crump WP, Steyn-Ross DA, Steyn-Ross ML, et al. An analysis of the transitions between down and up states of the cortical slow oscillation under urethane anaesthesia. J Biol Phys. 2010 Jun;36(3):245-59.

38. Haider B. Neocortical Network Activity In Vivo Is Generated through a Dynamic Balance of Excitation and Inhibition. J Neurosci. 2006;26(17):4535-45.

39. Chauvette S, Crochet S, Volgushev M, Timofeev I. Properties of Slow Oscillation during SlowWave Sleep and Anesthesia in Cats. J Neurosci. 2011 Oct 19;31(42):14998-5008. 
40. Kuo C-C, Bean BP. Na+ channels must deactivate to recover from inactivation. Neuron. April;1994(12):819-29.

41. Buffington SA, Rasband MN. The axon initial segment in nervous system disease and injury: The AIS in nervous system disease. European Journal of Neuroscience. 2011 Nov;34(10):1609-19.

42. Clark BD, Goldberg EM, Rudy B. Electrogenic Tuning of the Axon Initial Segment. The Neuroscientist. 2009 Dec 1;15(6):651-68.

43. Kuba $\mathrm{H}$, Oichi $\mathrm{Y}$, Ohmori $\mathrm{H}$. Presynaptic activity regulates $\mathrm{Na}+$ channel distribution at the axon initial segment. Nature. 2010 Jun 24;465(7301):1075-8.

44. Bender KJ, Uebele VN, Renger JJ, Trussell LO. Control of firing patterns through modulation of axon initial segment T-type calcium channels: Axon initial segment control of firing patterns. J Physiol. 2012 Jan 1;590(1):109-18.

45. Nelson SB, Turrigiano GG. Strength through Diversity. Neuron. 2008 Nov;60(3):477-82.

46. van Vreeswijk C, Sompolinsky H. Chaos in neuronal networks with balanced excitatory and inhibitory activity. Science. 1996;274(5293):1724.

47. Isaacson JS, Scanziani M. How Inhibition Shapes Cortical Activity. Neuron. 2011 Oct;72(2):23143.

48. Kaitin KI, Kilduff TS, Dement WC. Sleep fragmentation in canine narcolepsy. Sleep. 1986(1):116-9.

49. Prospéro-Garcia O, Herold N, Phillips TR, Elder JH, Bloom FE, Henriksen SJ. Sleep patterns are disturbed in cats infected with feline immunodeficiency virus. Proc Natl Acad Sci USA. December;1994(91):12947-51. 
50. Leemburg S, Vyazovskiy VV, Olcese U, Bassetti CL, Tononi G, Cirelli C. Sleep homeostasis in the rat is preserved during chronic sleep restriction. PNAS. 2010(36):15939-44.

51. Gorgoni M, Ferlazzo F, Moroni F, D’Atri A, Donarelli S, Fanelli S, et al. Sleep Deprivation Affects Somatosensory Cortex Excitability as Tested Through Median Nerve Stimulation. Brain Stimulat. 2014 Sep;7(5):732-9.

52. Huber R, Maki H, Rosanova M, Casarotto S, Canali P, Casali AG, et al. Human Cortical Excitability Increases with Time Awake. Cereb Cortex. 2013 Feb 1;23(2):1-7.

53. Jackson ML, Croft RJ, Owens K, Pierce RJ, Kennedy GA, Crewther D, et al. The effect of acute sleep deprivation on visual evoked potentials in professional drivers. Sleep. 2008;31(9):12611269.

54. Lim J, Dinges DF. Sleep Deprivation and Vigilant Attention. Ann N Y Acad Sci. 2008 May;1129(1):305-22.

55. Taheri M, Arabameri E. The effect of sleep deprivation on choice reaction time and anaerobic power of college student athletes. Asian J Sports Med. 2012;3(1):15.

56. Gal A, Eytan D, Wallach A, Sandler M, Schiller J, Marom S. Dynamics of Excitability over Extended Timescales in Cultured Cortical Neurons. J Neurosci. 2010 Dec 1;30(48):16332-42.

57. Ellingson RJ, Wilken K, Bennett DR. Efficacy of Sleep Deprivation as an Activation Procedure in Epilepsy Patients. Journal of Clinical Neurophysiology. 1984(1(1)):83-101.

58. Meisel C, Schulze-Bonhage A, Freestone D, Cook MJ, Achermann P, Plenz D. Intrinsic excitability measures track antiepileptic drug action and uncover increasing/decreasing excitability over the wake/sleep cycle. Proc Natl Acad Sci. 2015 Nov 24;112(47):14694-9. 
59. Scalise A, Desiato MT, Gigli GL, Romigi A, Tombini M, Marciani MG, et al. Increasing Cortical Excitability: A Possible Explanation For The Proconvulsant Role Of Sleep Deprivation. Sleep. 2006;29(12):1595-8.

60. Greene RW, Frank MG. Slow Wave Activity During Sleep: Functional and Therapeutic Implications. The Neuroscientist. 2010 Dec 1;16(6):618-33.

61. Davis CJ, Clinton JM, Jewett KA, Zielinski MR, Krueger JM. Delta Wave Power: An Independent Sleep Phenotype or Epiphenomenon? J Clin Sleep Med. 2011;Supplement to Vol. 7(5):S16-18.

62. Frank MG, Issa NP, Stryker MP. Sleep enhances plasticity in the developing visual cortex. Neuron. 2001;30(1):275-287.

63. Rial RV, Nicolau MC, Gamundí A, Akaârir M, Aparicio S, Garau C, et al. The trivial function of sleep. Sleep Med Rev. 2007 Aug;11(4):311-25.

64. Cirelli C, Tononi G. Is sleep essential? PLoS Biol. 2008;6(8):e216.

65. Xie L, Kang H, Xu Q, Chen MJ, Liao Y, Thiyagarajan M, et al. Sleep drives metabolite clearance from the adult brain. science. 2013;342(6156):373-377.

66. Wang G, Grone B, Colas D, Appelbaum L, Mourrain P. Synaptic plasticity in sleep: learning, homeostasis and disease. Trends Neurosci. 2011 Sep;34(9):452-63.

67. Tononi G, Cirelli C. Sleep and the Price of Plasticity: From Synaptic and Cellular Homeostasis to Memory Consolidation and Integration. Neuron. 2014 Jan;81(1):12-34.

68. Tononi G, Cirelli C. Sleep and synaptic homeostasis: a hypothesis. Brain Res Bull. 2003 Dec;62(2):143-50. 
69. Frank MG. Erasing Synapses in Sleep: Is It Time to Be SHY? Neural Plast. 2012;2012:1-15.

70. Frank MG. Why I Am Not SHY: A Reply to Tononi and Cirelli. Neural Plast. 2013;2013:1-3.

71. Peter-Derex L, Yammine P, Bastuji H, Croisile B. Sleep and Alzheimer's disease. Sleep Med Rev. 2015 Feb;19:29-38.

72. Ferrarelli F, Huber R, Peterson MJ, Massimini M, Murphy M, Riedner BA, et al. Reduced sleep spindle activity in schizophrenia patients. Am J Psychiatry. 2007;164(3):483-492.

73. Kundermann B, Hemmeter-Spernal J, Huber MT, Krieg J-C, Lautenbacher S. Effects of Total Sleep Deprivation in Major Depression: Overnight Improvement of Mood is Accompanied by Increased Pain Sensitivity and Augmented Pain Complaints. Psychosom Med. 2008 Jan;70(1):92-101.

74. Hemmeter U-M, Hemmeter-Spernal J, Krieg J-C. Sleep deprivation in depression. Expert Rev Neurother. 2010 Jul;10(7):1101-15.

75. Roizenblatt S, Neto NSR, Tufik S. Sleep Disorders and Fibromyalgia. Curr Pain Headache Rep. 2011 Oct;15(5):347-57.

76. Alberti A. Headache and sleep. Sleep Med Rev. 2006 Dec;10(6):431-7.

77. Irwin MR, Olmstead R, Carrillo C, Sadeghi N, FitzGerald JD, Ranganath VK, et al. Sleep Loss Exacerbates Fatigue, Depression, and Pain in Rheumatoid Arthritis. SLEEP. 2012 Apr $1 ; 35(4): 537-43$

78. Hengen KB, Lambo ME, Van Hooser SD, Katz DB, Turrigiano GG. Firing Rate Homeostasis in Visual Cortex of Freely Behaving Rodents. Neuron. 2013 Oct;80(2):335-42. 


\section{LEGENDS}

Fig. 1. Human surface EEG recorded from frontal electrode vs general reference. A. wake state: high frequency, low amplitude. B. Slow wave sleep: low frequency 0,5- $6 \mathrm{~Hz}$, high amplitude.

Fig. 2. Schematic diagram of a neuron, with dendrite trees, synapses, soma, axon hillock, axon initial segment and axon with myelinized segments och nodes. A dashed area denotes all synapses that contribute to synaptic strength. Another dashed area denotes the axon initial segment (AIS) that partially contributes to intrinsic excitability.

Fig. 3.

A. Cortical neurons with a consistently low excitability.

B. Cortical neurons with a consistently and sufficiently high excitability, which allows epileptic discharges to be propagated.

C. Corticala neurons with an average level of excitability as in B, but with a large distribution of neurons with both high and low excitability.

Fig. 4. Schematic drawing showing some levels of neuronal excitability A-C with possible consequenses.

A. Lower limit when generalized epileptic seizure can occur without provocation, all neurons calibrated to optimal level.

B. Optimal level for calibration.

C. Lower level when generalized seizure can occur with provocation such as electroconvulsive treatment, all neurons calibrated to optimal level.

Fig. 5. Some detailed description of examples of calibration and feedback in synaptic strength during the cyclical process in SWS and non-SWS, which show how synaptic strenght, post-synaptic excitability and FR during the process might iterate and finally approach their equilibrium values. The purpose of these examples is to show that some process at least is possible in stabilizing synaptic strength during calibration. Evolution might have found other and better processes. 
Fig. 6. Possible responses as a result of plasticity (e.g. after monocular depression):

A. Presumtive neural respons to stimulus on the conditions that corrective and suppressive feedback is absent.

B. Neural response to stimulus. Negative feedback suppresses the response. Response unchanged during the following sleep depression.

C. Neural response to stimulus, negative feedback suppresses the response. During the sleep that follows, as in this hypothesis, calibration restores the effect of negative feedback; therefore, the response reaches the same level as for A. Note the difference after sleep B-C, which is similar to the result of sleep consolidation.

Fig. 7. Widening of FR during non-sleep states corresponds to narrowing during the sleep state.

With the presumtion of normal distibuted FRs (other distributions give similar results)

$\mathrm{FR}_{\mathrm{a}}{ }^{2}=\mathrm{FR}_{\mathrm{b}}{ }^{2}+\mathrm{FR}_{\mathrm{W}}{ }^{2}$ where

$\mathrm{FR}_{\mathrm{a}}{ }^{2}$ is the variance in $\log \mathrm{FR}$ before the sleep period

$\mathrm{FR}_{\mathrm{b}}{ }^{2}$ is the variance in $\log \mathrm{FR}$ after the sleep period

$\mathrm{FR}_{\mathrm{W}} 2$ is the variance created in FR in individual pyramid cells during non-sleep periods.

The widening during non-sleep periods could thus be attributed to the variance in individual pyramid cells created in the non-sleep periods. 
Fig. 1

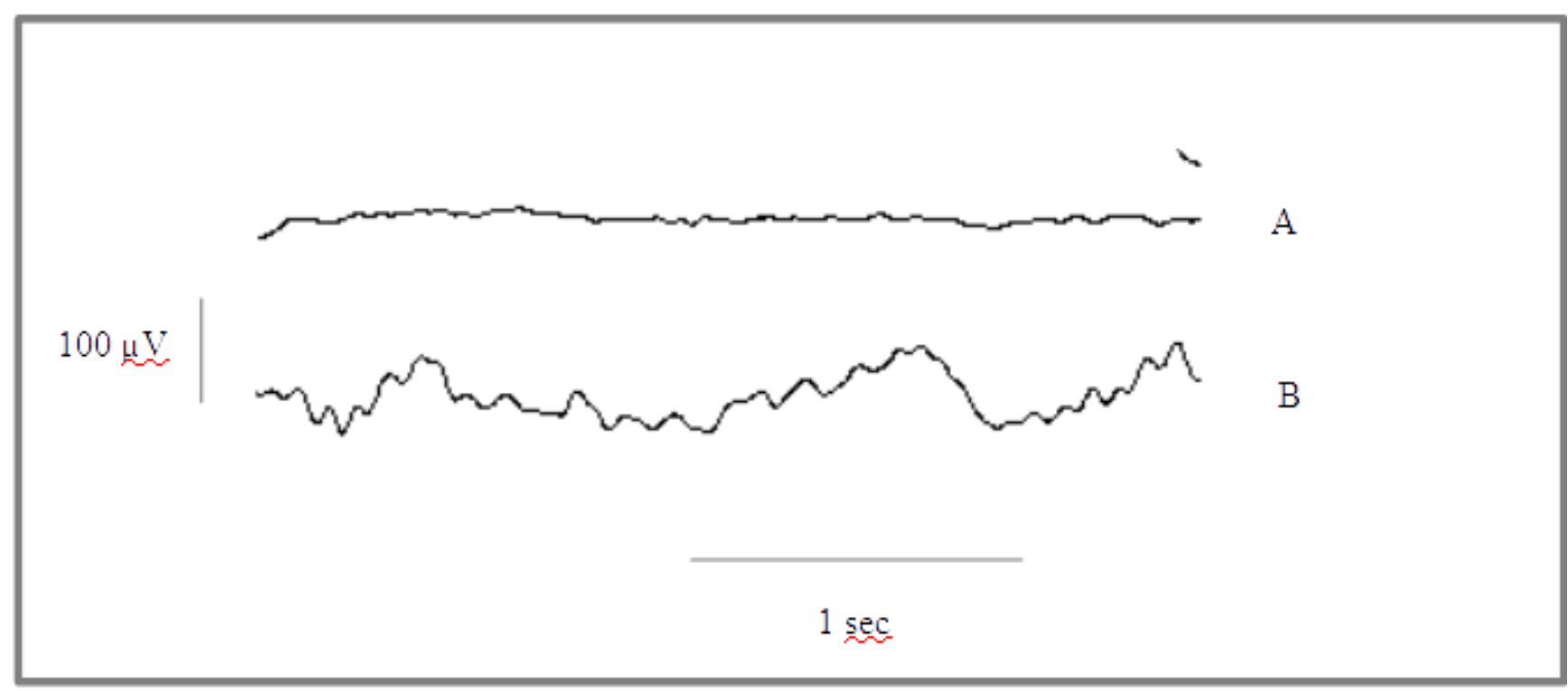

Fig. 2

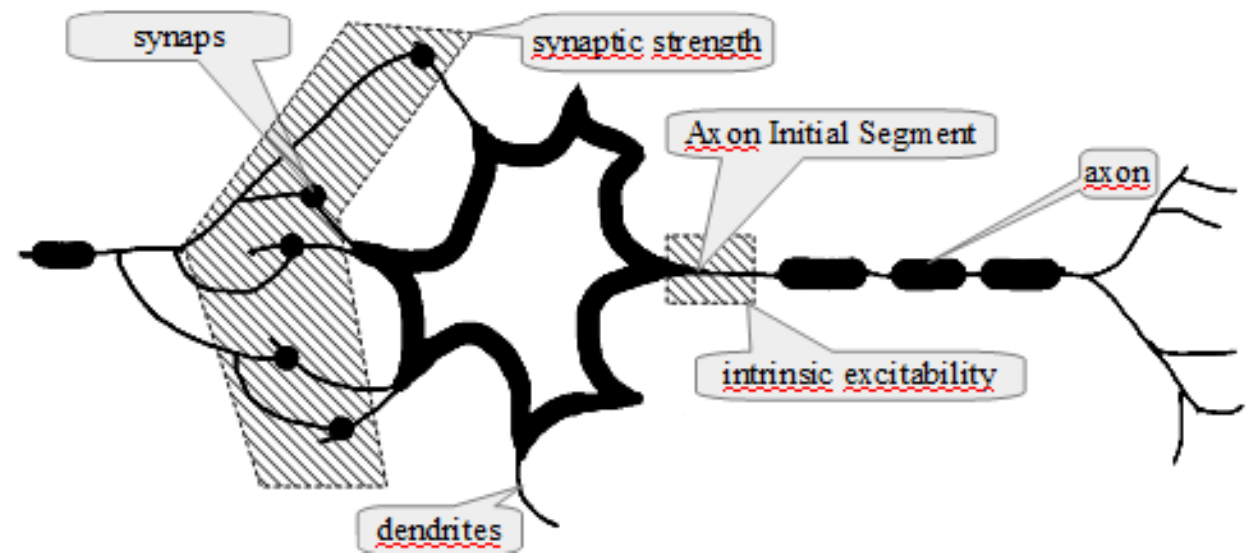


Fig. 3

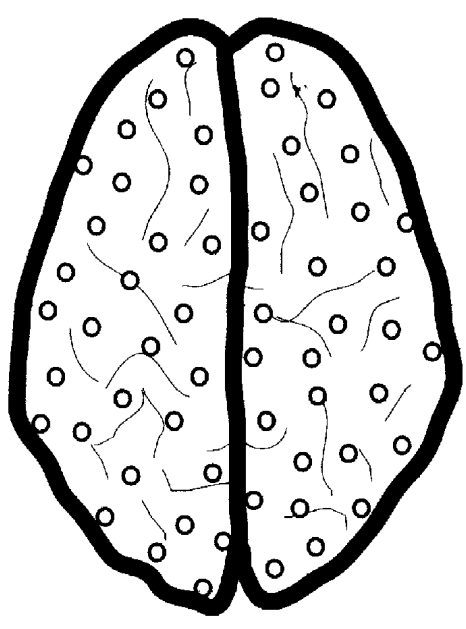

A

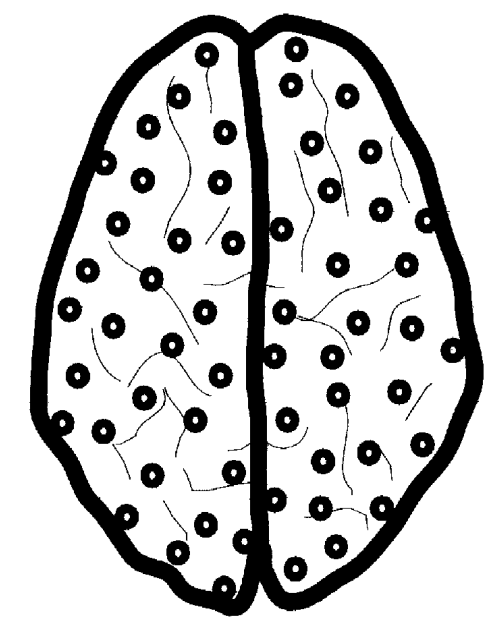

B

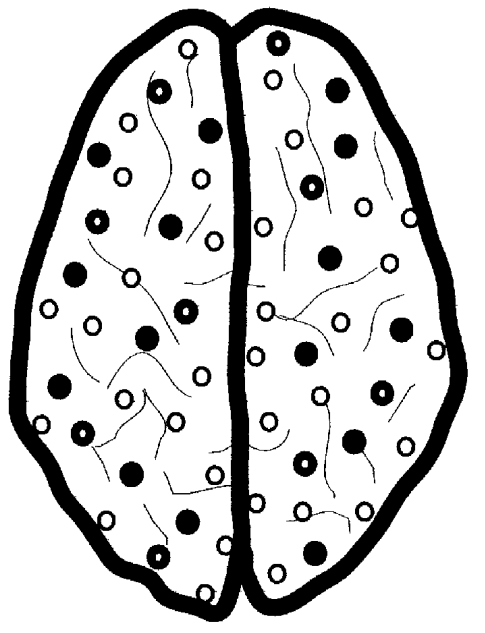

C

$$
\begin{aligned}
& \text { neuron with medium-high excitability } \\
& \text { neuron with high excitability } \\
& \text { neuron with low excitability }
\end{aligned}
$$

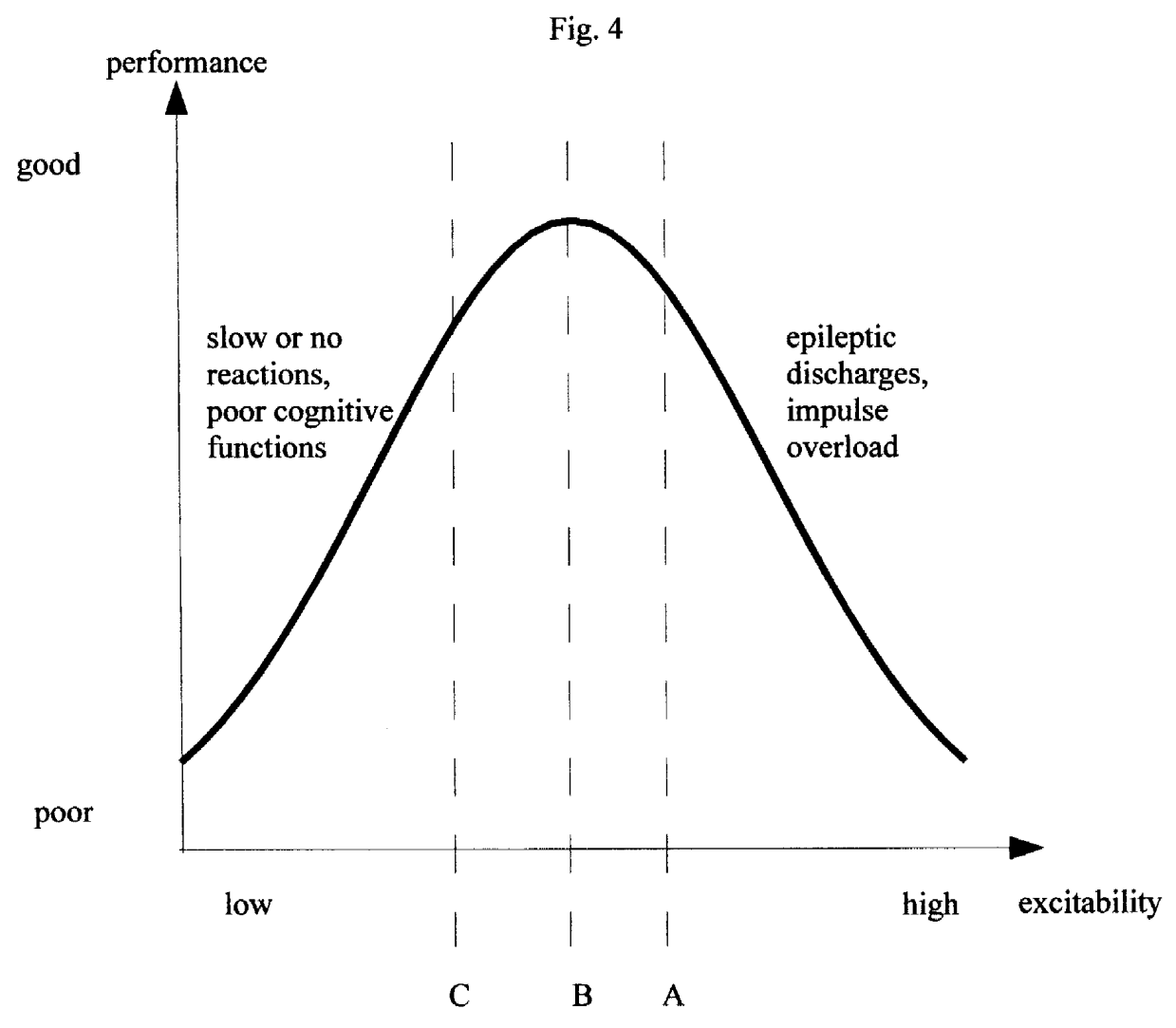



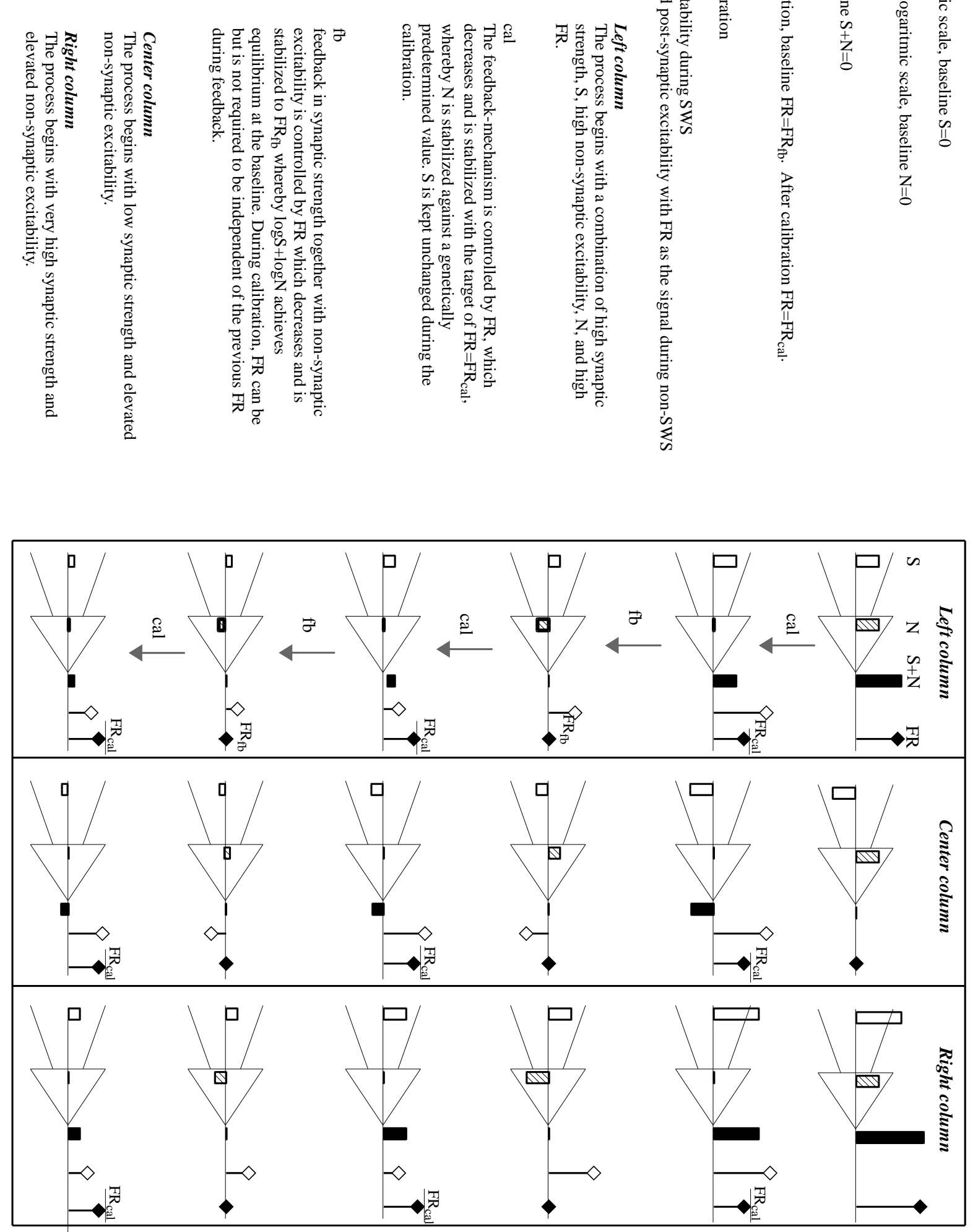
neuronal

response

high

Fig. 6.

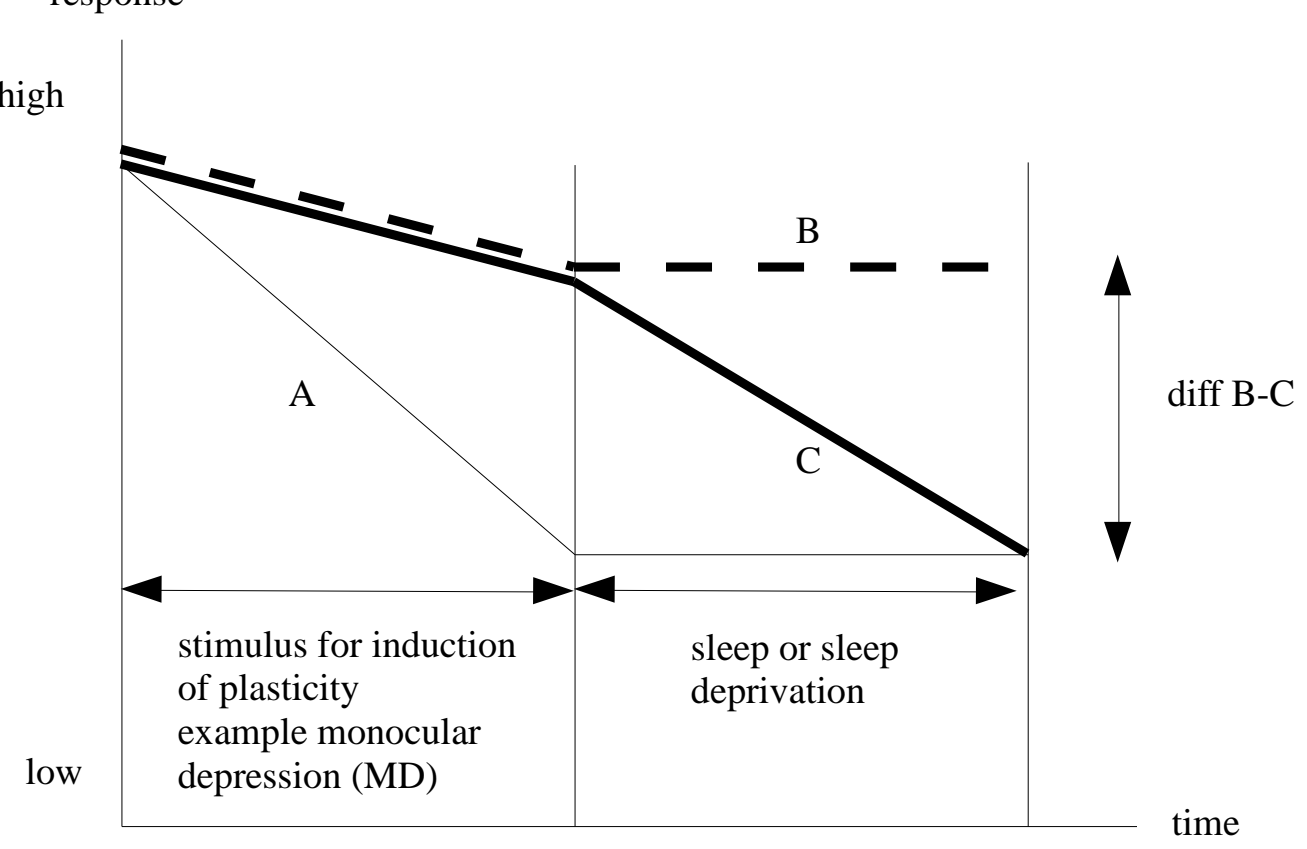

Fig. 7

nr

neurons

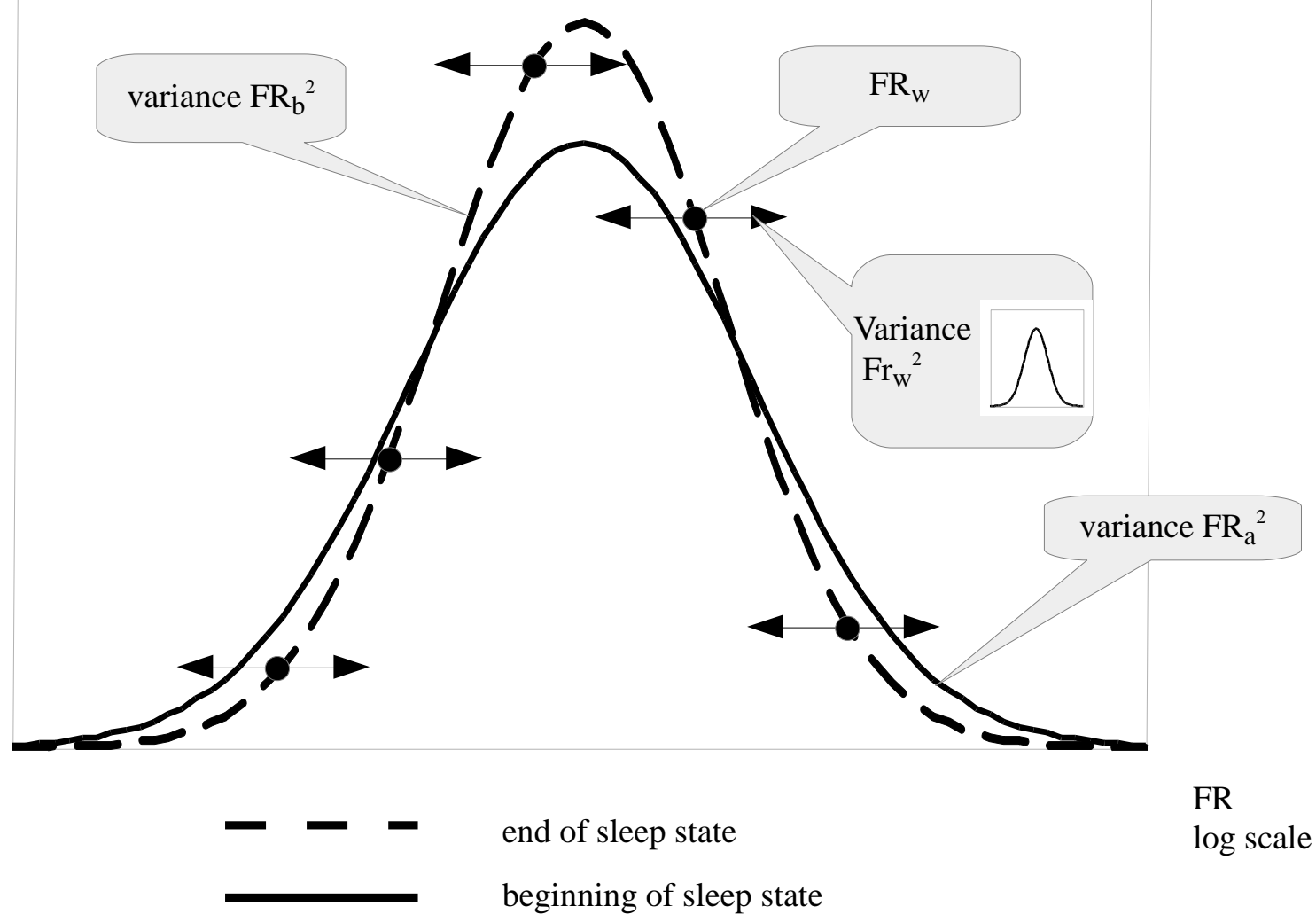

